\title{
La Dialéctica en la Psicologia del Desarrollo: Relevancia y Significacion en la Investigacion
}

\author{
Dialectic in Developmental Psychology: \\ Its Importance and Significance in Research
}

\author{
José Antonio Castorina* \\ Consejo Nacional de Investigaciones Científicas, Buenos Aires, Argentina \\ \& Universidad de Buenos Aires, Buenos Aires, Argentina
}

\begin{abstract}
Resúmen
El presente artículo examina la significación y la relevancia de la dialéctica en la obra psicológica de Piaget y de Vigotsky. Por un lado se analiza una metodología dialéctica común, presente en las unidades de análisis de las investigaciones. Por otro, se exponen los rasgos peculiares que adquiere la dialéctica en la explicación del desarrollo en ambos desarrollo. Finalmente, en base a los análisis realizados, se caracterizan los rasgos de la dialéctica. También se discute su unicidad y su carácter apriori en la Psicología del Desarrollo y en las Ciencias Humanas, justificándose su diversidad, en función de las temáticas que se indagan, así como a ciertos límites en el uso de esa categoría en la indagación empírica. Su implementación exige que adopte formas diferentes, pertinentes a los distintos problemas y focos teóricos. Palabras clave: Dialéctica, Unidad de Análisis, Contradicción, Inferencia Dialéctica, Equilibración.
\end{abstract}

\begin{abstract}
The present article analyses the signficance and relevance of dialectic in Piaget's and Vigotsky's psychological studies. On one hand, it highlights the common dialectical methodology present in units of analysis in research. On the other hand, it focuses on the peculiar features dialectic acquires within both authors' explanation of psychological development. Lastly, a characterization of dialectic features based on previous analysis is introduced. The present article argues against the unique dialectic and its $a$ priori features in Developmental Psychology and Human Sciences areas. It also justifies its diversity in relation to the subject matters under investigation as well as the existence of certain limits in the use of that category in empirical research. The use of dialectics requires the adoption of diverse forms regarding different issues and theoretical focuses.

Keywords: Dialectic; Unit of Analysis; Contradiction; Dialectic Inference; Equilibration.
\end{abstract}

La historia del pensamiento dialéctico exhibe una pluralidad de interpretaciones: desde el Taoísmo en la cultura china, Heráclito y Platón en la antigüedad clásica griega, pasando por Kant, Hegel y Marx, en el pensamiento moderno, luego los diferentes interpretaciones marxistas que incluyen el estructuralismo de Althusser, pasando por Gramsci, Colleti y Luporini, hasta la versión del open marxism de Holloway. Por otro lado, las revoluciones científicas en la física y la geometría de principios del siglo pasado suscitaron una renovada interpretación epistemológica de esta categoría en los términos de la interacción entre la teoría y la experimentación, entre lo dado y lo construido, lo apriori y lo aposteriori en las obras de Bachelard y de Gonseth (Castorina \& Baquero, 2005).
* Dirección: Universidad de Buenos Aires, Facultad de Filosofía y Letras, Instituto de Investigaciones en Ciencias de la Educación, Puán, $480,4^{\circ}$ Piso, Buenos Aires, Argentina, 1406.E-mail: iiceuba@gmail.com
Aunque a veces se ha postulado un denominador conceptual para todas las posiciones dialécticas, es preciso reconocer que cualquier caracterización general es inevitablemente imprecisa y demasiado general. Se ha buscado identificar un mínimo común denominador: el ocuparse del dinamismo y el movimiento provocados por las tensiones en un sistema conformado por relaciones entre los elementos de una totalidad. Los procesos dialécticos se han referido al despliegue del ser, a los fenómenos sociales o a la constitución del conocimiento. Centralmente, las diferentes versiones atribuyen la fuente del cambio a las interacciones propias del sistema en cuestión, por lo que se afirma su auto movimiento. Para la mayoría de las versiones, las contradicciones en el sistema constituyen la fuerza motriz de cualquier cambio dialéctico (Bidell, 1988).

No solo es sumamente difícil encontrar significados comunes entre las diferentes perspectivas filosóficas, también han sido cuestionadas las formas históricas que ha adoptado la dialéctica en la historia del pensamiento, 
muy particularmente en el pensamiento contemporáneo (Deleuze, 1970; Popper, 1967).

Desde otro nivel de análisis, se puede relevar la insatisfacción experimentada por la instrumentación de la categoría en la psicología y las ciencias sociales. Así, se la ha utilizado abusivamente "para pensar todo lo que ocurre en un campo determinado"; se la ha tratado como una solución puramente retórica a las dificultades que presenta una investigación: cualquier interacción intuida entre los componentes de una situación se ha calificado como "dialéctica", evitando precisar las relaciones en juego y su devenir. Para algunos críticos, dicha insatisfacción es insalvable por ser inherente a la dialéctica misma, en cualquiera de sus versiones, para otros es posible una instrumentación satisfactoria si el investigador atiende a las peculiaridades de los procesos de desarrollo en un campo determinado de conocimiento.

Nuestra discusión del alcance y los límites del pensamiento dialéctico se sitúa en el escenario de la psicología del desarrollo, examinando la elaboración de las teorías de Piaget y de Vigotsky. Sin desconocer su relevancia, no incursionaremos en los argumentos que se han esgrimido en la defensa o el rechazo de la dialéctica en cualquiera de sus versiones en la historia del pensamiento filosófico.

El examen de a dialéctica en Piaget y Vigotsky nos permite formular ciertas cuestiones más generales: ¿hay una única dialéctica o es diversa? ¿Es apriori o depende de la investigación teórica y empírica? ¿Es aplicable a cualquier problema o tiene límites? ¿Los conflictos se resuelven inevitablemente como han interpretado tantos psicólogos? ¿Cuál es su lugar en la explicación de los cambios del desarrollo? Tales interrogantes de la investigación psicológica no son ajenos a los formulado en otras ciencias sociales, por ello tenemos la esperanza de que estos análisis abran un diálogo interdisciplinario, apuntando a una renovación del pensamiento dialéctico (Lahire, 2006).

\section{El Problema de la Novedad}

La emergencia de la novedad en el desarrollo es el problema crucial de la psicología del desarrollo: en Vigotsky (1934/1993b), se refiere a la emergencia de las funciones psíquicas superiores como el lenguaje o la conceptualización, mediante la internalización de la cultura; en Piaget $(1978,1981)$, se refiere a la génesis de los sistemas de conocimiento que son irreductibles a los anteriores de los que provienen. Cabe recordar que la novedad interesó centralmente a Marx (1971), cuándo se ocupó de la génesis de la forma dinero en los intercambios de mercancías de un sistema económico.

Sin embargo, las corrientes dominantes en la actual psicología del desarrollo, se plantean las preguntas por las causas que han provocado el desarrollo ("Wich one?") por los "factores" que lo explican. Tales preguntas dependen de una estrategia intelectual, originada en el pensamiento moderno y que separa tajantemente los procesos considerados interiores al aparato mental del mundo exterior, la actividad individual de las determinaciones sociales, las condiciones naturales de las socio históricas (Overton, 1998). Se trata de un marco epistémico subyacente a los investigadores, algo así como su "sentido común" académico que no les ha posibilitado plantear adecuadamente la cuestión de la "novedad".

Las explicaciones psicológicas invocan las disposiciones y representaciones innatas para el desarrollo de los conocimientos, o las determinaciones sociales, cada una por su lado; incluso, se ha propuesto una sumatoria de aquellos factores. En su conjunto, tales explicaciones causales son por completo incapaces de dar cuenta de su emergencia. También las ciencias sociales testimonian las mismas dificultades para formular las preguntas y explicar la conformación de la subjetividad social, especialmente por la prevalencia obstaculizadora del pensamiento de la escisión entre individuo y sociedad (Bourdieu, 1988; Elias, 1983).

Por el contrario, la obra de Piaget y Vigotsky inauguró una perspectiva relacional en la psicología del desarrollo que integró en su movimiento a aquellos componentes de la experiencia con el mundo y fue luego prolongada y reformulada por otros teóricos (Boech, 1991; Thelen \& Smith, 1998; Valsiner, 2000). Un enfoque similar se encuentra, con sus propias peculiaridades, en el estructuralismo genético de la investigación social (Bourdieu, 1988; Elias, 1983) que articula dinámicamente al individuo y la sociedad, la materialidad y la representación simbólica. Estas teorías sociales y aquellas psicologías del desarrollo convergen con los enfoques contemporáneos "de sistema abierto" en la termodinámica, en la biología de la autorregulación, incluso en la teoría de Marx. En todos los casos, se trata de estudiar sistemas que están básicamente involucrados en relaciones de intercambio con sus medios particulares. Obviamente, cada sistema abierto implica una diferente noción de qué clase de relaciones de intercambio están implicados y cuáles son los objetos de tales intercambios. Mientras en un sistema biológico se trata de formas de intercambio de sustancias, los sistemas sociales dependen de las relaciones de los capitales económicos y de la utilización del "capital simbólico con su medio. En la psicología genética, los sistemas cognitivos interactúan entre ellos o con la información, hay intercambios entre los signos construidos socialmente que son comunicados e interpretados entre las personas, en la constitución de la vida psicológica. El interrogante central de cualquier teoría de sistema abierto es siempre de qué modo se transforman los estados estables alcanzados, como se reorganizan dichos sistemas de intercambio y se alcanzan otros nuevos.

El marco epistémico relacional es básicamente una estrategia de pensamiento que orienta la formulación de los problemas y las decisiones principales en el estudio 
de los sistemas sociales y psicológicos. Durante las investigaciones, dicho marco se convierte en una metodología dialéctica rigurosa para la elaboración teórica. Además, la dialéctica llega ser un componente central de una teoría explicativa para la producción de novedades en las ciencias sociales, planteada precisamente en los términos de la emergencia de estados de un sistema que se reorganiza.

\section{La Metodología Dialéctica}

El marco epistémico relacional orientó las investigaciones piagetianas sobre la adquisición de los conocimientos de las interacciones entre esquemas o entre ellos y los observables; también los estudios de Vigotsky referidos a la génesis de las funciones psíquicas superiores, en base a la interiorización individual de las herramientas culturales. Dicho marco posibilitó la metodología dialéctica para los problemas de la investigación atinentes a los procesos de desarrollo, la que no sustituye a los métodos específicos de la investigación empírica en Psicologia, sino que es un modo de construir la teoría. Se trata de una manera de formular los problemas en términos de la interrelación de los fenómenos que se estudian y de encarar una reorganización teórica de los resultados de la investigación científica.

De este punto de vista, tanto Piaget como Vigotsky siguieron el dictum de Hegel y de Marx según el cuál comprender un objeto de investigación equivale a ser consciente de la unidad concteta de las determinaciones contrarias que lo constituyen, en la totalidad de sus interrelaciones y de las contradicciones que lo constituyen.

Vigotsky, expícitamente, propuso una perspectiva dialéctica en psicología del desarrollo que desempeña el mismo rol del método científico seguido por Marx en El Capital para reconstruir la génesis de la mercancía dinero (Vigotsky, 1927/1991a). Es decir, el estudio genético de un proceso debe abordar el movimiento del objeto indagado en función de su historia y de la complejidad de sus nexos. Su principal problema era transferir este método a la investigación psicológica construyendo unidades de análisis. De este modo, un proceso de desarrollo podría ser estudiado en los términos de la unidad que contiene a los procesos y tendencias que le son propios; más aún, el autor intentaba identificar las relaciones y oposiciones que contribuyen a su producción (1927/1991a).

Así, Vigotsky diferenció en el estudio del lenguaje, el significado interno que es el aspecto semántico asociado a su exterioridad auditiva en los procesos de comunicación social, pero los consideró en sus interrelaciones. De este modo, la unidad del lenguaje no es homogénea sino compleja, consiste en relaciones que evitan el aislamiento de los componentes internos y externos, del significado social e individual de la palabra. La unidad de análisis incluye en sí los procesos relevantes que originan la existencia del objeto estudiado. El significado de la palabra incluye relaciones y contradicciones, tanto las tensiones entre lo externo y lo interno, como entre el individuo y la sociedad (Vigotsky, 1934/ 1993b). En el caso de los sistemas psicológicos, como unidades más amplias de análisis para el estudio del desarrollo, busca elaborar la delicada interacción de los aspectos naturales y culturales, fisiológicos y psicológicos, individuales y sociales. Nuestro autor intenta atrapar las interacciones entre niveles exteriores e interiores del funcionamiento psicológico, sin renunciar a la complejidad propia de cada uno de ellos (Vigotsky, 1930/1991b).

Piaget, por su lado, estudió los problemas del desarrollo cognoscitivo abordando las relaciones constitutivas del fenómeno, caracterizándolas en los términos de sus interacciones recíprocas. Así, al plantear un problema teórico trataba de articular la heterogeneidad inicial de las funciones o las propiedades predicadas, introduciendo su vinculación sistemática. El procedimiento principal consistió en integrar paso a paso los elementos heterogéneos en una configuración de relaciones bipolares, una unidad totalizadora de los componentes opuestos, a los fines de reconstruir el dinamismo de la formación de los conocimientos. Al estudiar la génesis de los sistemas operacionales, trató las relaciones bipolares entre el sujeto y el objeto, la asimilación y la acomodación, las acciones y las significaciones, las estructuras y los procedimientos, entre la abstracción empírica y la abstracción reflexionante. Estas relaciones son las unidades de análisis que involucran los aspectos contrarios o unidades dinámicas de contrarios.

Así, para dar cuenta del proceso cognoscitivo en la primera infancia (1969) elaboró una argumentación teórica que articula en una relación constitutiva a las funciones opuestas de asimilación y acomodación (1969) o para producir una definición genética de la inteligencia integró dinámicamente a través de las diferentes estructuraciones el carácter propiamente estructural o dis-continuo de la actividad y su funcionamiento continuo (1970). Al final de su obra, estudió la construcción y el uso de las estructuras matemáticas por los sujetos, examinando cómo éstos ponían en juego procedimientos "heurísticos" para aplicarlas en situaciones concretas, y a la vez cómo las estrategias utilizadas para resolver un problema práctico con objetos involucraba una estructura que debe ser transferida de "una situación a otra". Se trataba de comprender la interdependencia de las estructuras y los procedimientos en sus interacciones y aún en sus oposiciones (Bidell, 1988; Inhelder \& Piaget, 1979).

En las ciencias sociales hay un enfoque metodológico semejante (Bourdieu \& Wacquant, 2005): los dilemas o el antagonismo aparentemente irresoluble entre las estructuras sociales y la agencialidad se superan con un método que reconsidera el movimiento de una realidad intrínsecamente doble del mundo social. 


\section{La Dialéctica del Desarrollo}

Piaget caracterizó los procesos dialécticos en la formación de los conocimientos en base a estudios experimentales específicamente dirigidos a este fin (Piaget, 1978, 1981).

En primer lugar, las contradicciones naturales, a diferencia de las contradicciones lógicas, surgen a propósito de problemas que el sujeto se plantea y no puede resolver previamente. Por ejemplo, un niño que intenta explicar el equilibrio de una balanza donde los pesos se desplazan sobre una barra, piensa en el factor peso por separado y se ve envuelto en contradicciones. Para él, una misma acción puede resultar en efectos diferentes, ya que al colocar un mismo peso, éste puede subir y bajar el peso, aunque normalmente baje; o una acción y su contraria no dan lugar a compensaciones completas: al añadir pesos a un platillo que ya tiene otros se lo hace bajar, pero también puede ocurrir lo mismo si se los quita. En general, él no poder pensar la articulación de las propiedades físicas provoca las contradicciones. Ulteriormente, el niño llega a interpretar el problema en términos del "momento" de los pesos con respecto del centro, y no solo de cada peso, por una progresiva relativización de la comprensión del concepto de peso y por un incremento de su referencia. De este modo, un mayor peso a menor distancia se puede compensar con otro menor a mayor distancia del centro (Piaget, 1978).

Cualquier contradicción se explica por el carácter incompleto de las compensaciones entre afirmaciones (atribuir una propiedad $a$ un clase A) y las negaciones (atribuir no- $a$ a la clase complementaria A') Dada la inmediatez de las afirmaciones, las dificultades en articularlas con sus negaciones son la causa de de las contradicciones del pensamiento natural.

Sin embargo, las contradicciones no son el motor del desarrollo cognoscitivo, sino más bien una instancia del proceso más amplio de equilibración de los sistemas de conocimiento. La reorganización de tales sistemas se explica, en primer lugar, por las desequilibraciones entre afirmaciones y negaciones, las que provocan los conflictos; pero más tarde se activan los procesos de reequilibración que compensan las afirmaciones con las negaciones, por medio de las abstracciones reflexionantes e invención de nuevos posibles. Las contradicciones forman parte de un proceso de conjunto que involucra a mecanismos constructivos que producen la "novedad" cognoscitiva.

En segundo lugar, en Las Formas Elementales de la Dialéctica (1981) reconsidera a la dialéctica en términos de una inferencia -no deductiva- que lleva de un sistema conceptual a otro más avanzado, en el sentido de sus relaciones lógicas, irreductibles a las anteriores (por ejemplo, una conservación lógica respecto a las hipótesis de no conservación). Básicamente, Piaget pensaba en niveles de creciente complejidad sin preformación o arbitrariedad, un "lado inferencial de la equilibración", en tanto un nivel de significación se transforma en otro. Dicho inferencia se corresponde con la interacción sistémica de las acciones que produce la novedad cognoscitiva. Esta actividad inferencial presenta las siguientes modalidades: una aproximación indefinida del sujeto al objeto, por la interacción de la asimilación y la acomodación; una articulación de subsistemas que no han estado vinculados anteriormente; el pasaje de una indiferenciación inicial de propiedades a su diferenciación e integración conceptual; la transformación de una propiedad antes considerada absoluta a otra que se concibe dentro de un sistema; la construcción de un sistema más avanzado que da lugar a la reorganización de uno anterior, en un juego de proacción y retroacción.

Demos un ejemplo del proceso de diferenciación e integración, quizás la más relevante de las modalidades inferenciales para estudiar la génesis del conocimiento. Un niño pequeño que observa de frente un bloque de montañas, imagina que si lo viera del lado opuesto, sería tal como lo ve de frente. Es decir, los puntos de vista permanecen indiferenciados. Solo progresivamente llega a diferenciar los puntos de vista, hasta construir un sistema operatorio de perspectivas que permite considerar simultáneamente la reciprocidad de las relaciones en juego. Se puede hablar de un proceso inferencial que va desde la indiferenciación de las propiedades hacia la diferenciación e integración de los puntos de vista.

Hay confirmaciones parciales de esta dialéctica inferencial en estudios sobre cambio conceptual en el dominio biológico (Carey, 1999), ya que niños entre 4 y 10 años consideran inicialmente al concepto de ser vivo a partir de la mezcla de las propiedades de lo que es real, activo y existente; lo no vivo es para ellos no estar vivo, es decir no tener actividad - dada su relación con el comportamiento -, no existir ni estar presente a la mirada. Por un proceso de diferenciación, aquel concepto indiferenciado de vida se convierte en un concepto biológico intuitivo y el concepto indiferenciado de no vivo se convierte en el concepto de muerte. Ambas nociones se han reestructurado progresivamente respecto del animismo, en una biología del ciclo vital que atribuye una función a las partes internas del organismo y a las sustancias que se mueven alrededor del organismo.

La escuela socio-histórica (Vigotsky, 1934/1993b) concibe el desarrollo del niño como un complejo proceso caracterizado por la periodicidad, la novedad en la aparición de diferentes funciones, así como la metamorfosis o transformaciones cualitativas de una forma en otra, incluido el entrelazamiento entre la línea natural y la línea cultural del desarrollo. Las funciones psicológicas superiores son tanto sociales como individuales, ya que los niños llegan a dominar las herramientas culturales mientras interactúan con otro que sabe más. Estas adquisiciones solo existen en la relación de los opuestos (la transmisión cultural y la actividad del individuo) y la hegemonía de cada polo antagónico se modifica hacia el otro durante su génesis. 
Para el psicólogo ruso, el desarrollo de las funciones psicológicas superiores sigue una dialéctica propiamente hegeliana del en si, fuera de sí y para sí. A modo de ejemplo, al estudiar el movimiento interrumpido o no exitoso del bebé que pretender alcanzar un objeto se verifica que éste pasa a ser interpretado por otra persona como un gesto indicativo. Finalmente, dicho bebé llega a relacionar su fracasado movimiento con toda la situación y considera su movimiento como una indicación para que le alcancen el objeto. Es decir, pasa a ser sí mismo a través de los otros.

Lo anterior constituye la esencia del proceso de desarrollo cultural: "La personalidad viene a ser para sí lo que es en sí, a través de lo que significa para los demás" (Vigotsky, 1931/1993a, p. 149). Esta secuencia, que incluye la unidad de los contrarios, está despojada de la mística hegeliana y tiene pleno sentido para dar cuenta de la conformación de la subjetividad desde el punto de la apropiación de la cultura.

Finalmente, al establecer nuestro autor la doble naturaleza de los pseudoconceptos, que sirven como conexión entre el pensamiento por complejos y los conceptos artificiales, pone de relieve la creación de conceptos en el niño: "Debido a la contradicción inherente a él, mientras es un complejo, ya contiene el núcleo de un concepto futuro, el cuál se está desarrollándose en su interior" (1994, p. 232). Sin embargo, la contradicción no se resuelve por una superación inherente a la propia dinámica cognitiva individual o del propio concepto complejo sino que se dirime en los procesos de interacción social que presumen la existencia de los procesos de pensamiento en complejos, sin que éstos, a su vez, se reduzca a procesos sociales. "Es decir, coexiste una dualidad de procesos sociales e internos ..." (Castorina \& Baquero, 2005, p. 191). El resultado es la síntesis de la contradicción entre los sistemas de significado sociales y los procesos de apropiación del sujeto, lo que constituye una especie de unidad funcional durante el desarrollo. Utilizando nuevamente el lenguaje hegeliano:

En el niño, concepto en sí y para otros se desarrolla antes que para sí mismo. El concepto en sí y para otros está ya presente en el pseudos concepto y es el prerrequisito genético básico para el desarrollo de conceptos en el verdadero sentido del término. (Vigotsky, 1994, p. 232).

Estos son estrictamente momentos "teóricos" que no equivalen a una secuencia lineal o unívoca de adquisición que va de los pseudoconceptos a los conceptos. Más bien, el desarrollo es un proceso de coexistencia y desencuentro entre las formas conceptuales en las interconexiones sociales, lo que hace pensar en un curso relativamente incierto.

\section{Interpelando a la Dialéctica}

El estudio de las teorías de Piaget y de Vigotsky parece mostrar que la utilización de la categoría resulta perti- nente para elaborar los problemas de la emergencia de novedades. Ahora bien, luego de los análisis anteriores volvemos a los interrogantes planteados en la Introducción para responderlos: la dialéctica en la investigación psicológica ¿es una categoría a priori?; ¿Hay una sola manera de concebir sus rasgos? ¿el pensamiento dialéctico tiene límites?; ¿Las contradicciones se resuelven inevitablemente? ¿Las contradicciones son suficientes para explicar el cambio psicológico?

\section{Contra la Dialéctica Apriori}

Como se ha dicho, el carácter especulativo y forzado del empleo de la dialéctica en el conocimiento social plantea la cuestión de si es posible legitimarla. En nuestra opinión, el análisis de la dinámica de las interacciones entre los componentes de un sistema psicológico, en términos de contradicciones o de términos opuestos, a la manera de Vigotsky, o la dialéctica inferencial a la manera de Piaget, es inconcebible sin una interpretación sistemática de investigaciones teóricas y empíricas.

En ambos autores, la dialéctica fue básicamente una perspectiva metodológica y luego formó parte de una teoría del desarrollo, la que se atiene a su vez a determinados requisitos empíricos. Esta dialéctica no fija el "debe ser" de la investigación, no deriva simplemente del pensamiento filosófico, ni es una "aplicación" de este último a los fenómenos psicológicos.

Lo importante desde el punto de vista epistemológico es buscar la consistencia entre el marco epistémico dialéctico, su formulación metodológica y la teorización en el campo de la psicología del desarrollo. La eliminación de una dialéctica apriori constituye la lección principal de la obra de Piaget y de Vigotsky.

$\mathrm{Al}$ invocar esta categoría se debe tomar en cuenta un cierto objeto de conocimiento construido por el investigador, sea la reorganización de los conceptos o de sistemas lógico-matemáticos, la formación del lenguaje. Se requiere de una teoría específica acerca estos procesos, de modo tal que su reconstrucción se pueda caracterizar como dialéctica, porque de lo contrario ésta se impondría desde el exterior al campo de los fenómenos que se investigan.

La investigación teórica en psicología le otorga un lugar destacado a los procesos dialécticos, pero la credibilidad de esta hipótesis se sostiene indirectamente con la verificación de las afirmaciones sobre la génesis de los procesos psíquicos superiores o la génesis de los sistemas de conocimiento. Más aún, las virtudes o las insuficiencias de una dialéctica del desarrollo dependen de su consistencia con la teoría psicológica de conjunto y de si cumplen con los motivos que llevaron a postularla.

\section{Por la Diversidad}

Nuestro análisis ha mostrado que la formulación dialéctica del desarrollo se adecúa a la dinámica del campo específico de investigación que se ha recortado, y según la naturaleza de los problemas planteados. 
Así, es discutible que se pueda utilizar adecuadamente la dialéctica hegeliana para describir la constitución de la noción de peso, las proyecciones geométricas, o el número, estudiados por Piaget. No tiene sentido decir de esos conceptos "que se engendran a sí mismos" por medio del trabajo de lo negativo que lleva a otros conceptos, o que "se despliegan hacia formas superiores". Tampoco lo tiene en la historia de la ciencia sostener que la teoría de Newton se "niega" a sí misma para alcanzar la física relativista. En otras palabras, no hay una actividad interna a los propios conceptos en la construcción individual de las ideas y de las teorías científicas, las negaciones no se producen "desde dentro" de los conceptos.

La actividad constructiva se asemeja a la "negación externa" que da lugar a una generalización dialéctica, en el sentido de Bachelard: la actividad del "no" es una construcción que produce la síntesis que engloba los aspectos inconciliables o que generaliza lo negado. Claramente, está involucrada una actividad del sujeto que integra una estructura más débil en otra más rica o que produce las diferenciaciones e integraciones a partir de propiedades anteriores.

Por otra parte, sería absurdo decir que la suma es contradictoria con la resta y su articulación supera la contradicción para constituir un sistema operatorio. Podemos afirmar que dichos conceptos son construidos por el sujeto de conocimiento mediante un proceso, e incluso donde juegan un cierto papel las contradicciones, como se dijo antes. Pero aquí la "unidad de los contrarios" es un logro del proceso constructivo, de las inferencias que articulan sistemas de origen independiente entre sí.

Para Vigotsky, los fenómenos psíquicos superiores se sostienen en la contradicción "interna" entre el individuo y la sociedad, o los procesos interpsicológicos e intrapsicológicos, aún en la elaboración de los conceptos artificiales, en tanto son polos opuestos de la apropiación psicológica de las herramientas culturales. Aquí sí tiene sentido decir que la vida psicológica superior tiene como motor a la contradicción de los términos mencionados. Incluso, se puede afirmar que el psiquismo superior sólo existe en la relación de los opuestos, y en la modificación de la hegemonía de uno de los polos antagónicos hacia el otro durante la génesis. De este modo es válida la terminología hegeliana del en sí, el fuera de sí y el para sí para caracterizar la apropiación psicológica de las herramientas culturales o la interacción de los rasgos idiosincrásicos y los sociales de la adquisición conceptual.

En resumen, la dialéctica del desarrollo en Piaget y en Vigotsky no exhibe un único conjunto de reglas ni de leyes dialécticas. Sus rasgos son específicos al campo de fenómenos psicológicos bajo estudio, estamos ante una diversidad de formas dialécticas según se consideren la génesis de los conocimientos matemáticos a partir de otros anteriores o la formación del lenguaje o de los conceptos en base a los antagonismos entre actividad individual e interacción social. Habría que preguntarse si en otros campos disciplinarios de las ciencias sociales sucede algo semejante.

\section{Los Límites a la Dialectización}

¿La dialéctica se aplica a cualquier situación o tiene validez solamente para determinadas fases de un proceso de desarrollo?

Básicamente, no es una pura forma o una cáscara vacía que pudiera aplicarse a cualquier objeto o a cualquier situación (Grüner, 2006). En primer lugar, en Marx (1971) la contradicción dialéctica no sustituye ni se confunde con la contradicción lógica. Así, por un lado, cuestionó la inconsistencia de ciertos textos clásicos de economía porque afirmaban y negaban al mismo tiempo una cierta propiedad; por el otro, al tratar el movimiento social atribuyó pleno sentido a la contradicción dialéctica, ya que no se afirma y niega la misma cosa de la misma entidad, en el mismo tiempo, sino en un proceso. De modo semejante, Vigotsky no confundió la contradicción dialéctica que era imprescindible para dar cuenta de la génesis de los procesos psíquicos superiores con la contradicción lógica o las incoherencias lógicas que denunció en los psicólogos de su tiempo.

En el caso de Piaget, las contradicciones naturales que se producen durante el choque de los observables y los esquemas o entre estos últimos ante las situaciones problemáticas no se confunden con las contradicciones lógicas, que tienen sentido dentro de un sistema de enunciados. Estas últimas se corrigen revisando las premisas del razonamiento, mientras que las primeras se "superan" porque dan lugar a un conocimiento más avanzado. Por otra parte, las contradicciones naturales y las inferencias dialécticas son propias de la fase de estructuración de los sistemas de conocimiento, del proceso de reorganización de dónde emergen las novedades. Por otro lado, en la fase de estabilidad de dicho proceso de equilibración, los sistemas de conocimiento que caracterizan a esos estados de relativo equilibrio cognoscitivo se rigen por inferencias deductivas, al igual que las teorías científicas. Se asiste a una alternancia entre el análisis formal de los sistemas conocimiento y la reconstrucción de su proceso de reorganización (García, 2002).

Por último, Marx había sugerido que las categorías propias de una presentación dialéctica son específicas para un área particular bajo estudio. No hay, ni puede haber, un sistema dialéctico que sea de aplicación universal, ya que es relativo esencialmente al contexto global de las condiciones de producción material en un determinado momento histórico. En el pensamiento de Piaget la dialéctica correspondía exclusivamente a la praxis humana, especialmente a la construcción de los conocimientos, no podría hablarse de contradicciones ni de inferencias por fuera de ella. Para Vigotsky, la internalización dialéctica de la cultura en un juego de opuestos transcurre solo si se dispone de determinadas herramientas socialmente producidas y de una actividad 
individual de apropiación, bajo ciertas condiciones contextuales.

Sin embargo, Vigotsky siguió en ocasiones las tesis de Engels y Lenin según las cuáles habría una universalidad de las "leyes" dialécticas, para el pensamiento, la naturaleza y la sociedad. En las discusiones de los años setenta, y que hoy pueden resultar algo anacrónicas, García (1970) cuestionó la dialéctica de la naturaleza porque sería inconsistente con la tesis central de la construcción dialéctica de la ciencia al colocar la objetividad como ya dada en el punto de partida del conocimiento (el mundo se refleja en el conocimiento). Además, la dialéctica interpretada como un método universal aplicable a todo fenómeno sería una abstracción divorciada de la exigencia del análisis concreto de la praxis humana, en una cierta sociedad y en un momento histórico determinado, exigida por Marx.

\section{La Resolución de los Conflictos}

La crítica contemporánea a la dialéctica se ha dirigido contra el carácter forzoso de las superaciones de las contradicciones o el progreso inevitable que conllevaba (Castorina \& Baquero, 2005; Deleuze, 1970), justamente por eliminar la producción de la novedad.

Marx puso de pie a la dialéctica hegeliana, caracterizada por ser el despliegue de una unidad espiritual originaria que debe realizarse, proponiendo una tensión constitutiva entre los términos opuestos en su unidad, a la que corresponde una serie de posibles resoluciones, pero ninguna ha de suceder inevitablemente. Las condiciones de la historia actual pueden llevar a realizarlas o a frustrarlas. Por otra parte, dadas las condiciones que pueden favorecer la realización de una determinada posibilidad, se dará una línea u otra de desarrollo social.

En buena parte de la obra de Piaget, el pasaje de un sistema de conocimiento tenía que ver con una tendencia al equilibrio de los sistemas de conocimiento, un inmanentismo explicativo que disminuyó el espíritu dialéctico que había presidido su metodología. Incluso, los conflictos cognitivos parecían resolverse para Piaget "tarde o temprano", atribuyendo una linealidad al desarrollo de los conocimientos. Sin embargo, la perspectiva de sistema complejo (García, 2002) permite renovar la teoría piagetiana del desarrollo, situando a las novedades que emergen del funcionamiento del subsistema cognoscitivo en un proceso de múltiples interacciones entre los subsistemas involucrados (subsistema biológico, cognitivo y social). Particularmente, la intervención de condiciones sociales "de contorno" que limitan y posibilitan la construcción conceptual permite afirmar una cierta incertidumbre en la psicogénesis (García, 2002). De este modo, podría no haber conflictos cognoscitivos o no ser resueltos por ausencia de las situaciones contextuales que lo posibilitaran; incluso, aquellas condiciones socio culturales pueden favorecer una línea de desarrollo en lugar de otra. Claramente, en un sistema abierto de interacciones, un estado de equilibrio en el sistema de conocimiento no determina unívocamente su reorganización. Más aún, es factible pensar en múltiples caminos de elaboración, según las interacciones del sistema de conocimiento con sus condiciones sociales.

Para Vigotsky, los sistemas de oposiciones entre la actividad intersubjetiva e intrasubjetiva no se transforman de modo inevitable ni se orientan en una misma dirección. El carácter estrictamente contextual de las relaciones entre herramientas culturales y los individuos que se apropian de ellas da lugar a la indeterminación de las transformaciones. El acoplamiento o la integración de los sistemas de actividad del niño y los instrumentos culturales "produce una serie que no es única y continua a cal y canto, sino a una serie de género, de índole y grados diferentes" (Vigotsky, 1994). Incluso, la diversidad e incertidumbre se pone de manifiesto en los sesgos del desarrollo psicológico con su propia cualidad en los estudios sobre niños ciegos o sordos según los entrecruzamientos del sistema de regulación natural y el sistema de regulación social. Como se ha dicho, el desarrollo se muestra abierto e incierto, ofreciendo una diversidad que no resulta de un desarrollo canónico. Sin embargo, en Vigotsky, como en Piaget, se observa una versión "progresista" del desarrollo orientado hacia el conocimiento científico, lo que es desmentido por los estudios más proclives a la diversidad (Castorina \& Baquero, 2005).

\section{La Dialéctica y la Explicación del Desarrollo}

Finalmente, ¿cuál es el lugar de la dialéctica en la explicación de las novedades?

Las explicaciones "sistémicas" del desarrollo psicológico ponen el énfasis en el intercambio de los sistemas psicológicos con sus medios particulares, los que son específicos para cada campo del desarrollo. En estos intercambios se ponen en juego la unidad de contrarios, las contradicciones, las superaciones, o las inferencias de nuevos conceptos.

La explicación de la génesis de los procesos superiores en Vigotsky, desde el lenguaje hasta los conceptos, trata de atrapar el despliegue histórico de los momentos centrales de aquellos procesos, poniendo de manifiesto sus nexos dinámico-causales. Como en Marx, la dialéctica en los términos de las contradicciones es el corazón del proyecto de dar cuenta de la emergencia de novedades. La unidad de los componentes opuestos de un sistema de funcionamiento psicológico, sus tensiones y su síntesis superadora, son la causa de la transformación psicológica. Así, la explicación psicológica busca alcanzar las síntesis o la unidad dialéctica que conserva las tensiones entre las líneas natural y cultural del desarrollo en la ontogénesis, atendiendo a la naturaleza cualitativa de los cambios.

En cuánto a la explicación piagetiana, las contradicciones no son suficientes por sí mismas para generar la reorganización de los sistemas conceptuales, constituyen una instancia del proceso más amplio de equilibración. 
Para la producción de novedades se requiere de los conflictos, pero luego se requiere de las abstracciones reflexionantes y las inferencias dialécticas que llevan hacia los estados más avanzados de conocimiento, no contenidos en las premisas. Se apela a la reorganización de los sistemas de conocimiento a través de las interacciones del sujeto y el objeto en diversos dominios. En todo caso, la contradicción es solamente un componente del mecanismo explicativo de la construcción cognoscitiva. Por su parte, la dialéctica inferencial es el lado "significativo" del mecanismo explicativo de la equilibración, y su contribución al desarrollo cognoscitivo reside en articular la novedad y la continuidad, las condiciones previas de un sistema conceptual y la indeterminación del pasaje de un sistema a otro.

\section{El Significado de la Dialéctica}

De lo expuesto se deriva una conclusión para los psicólogos del desarrollo: la dialéctica puede ser una herramienta válida solamente si no se supone que todos los campos y dimensiones de estudio siguen el mismo decurso y deben ser pensados del mismo modo. Se ha mostrado que la dialéctica adopta diferentes formas teniendo en cuenta el recorte de los problemas y la construcción de los objetos de investigación. Incluso, algunas son muy originales respecto del pensamiento dialéctico clásico, como sería el caso de las inferencias no deductivas en las fases estructurantes de los conocimientos. En este sentido, la tesis de la ortodoxia marxista según la cuál sólo se puede utilizar legítimamente la dialéctica si se recurre a las contradicciones "que mueven al mundo" es un retorno a la pura especulación filosófica.

Hemos argumentado que la reconsideración de la dialéctica es posible si no la consideramos como un capital ya disponible para ser utilizado "como está"; si nos negamos a que todos campos de estudio siguen el mismo decurso y bajo las mismas leyes; si admitimos que puede adoptar diferentes figuras teniendo en cuenta las dimensiones de los fenómenos que se indagan en cada disciplina o enfoque teórico.

Por otra parte, los investigadores de la psicología y de las ciencias humanas tienen que revalidar las credenciales de la dialéctica, estableciendo si como metodología es fructífera en dar lugar a un conocimiento original y bien fundado en un campo de estudio; evaluando si la dialéctica que se conjetura es consistente con la teoría específica de ese campo, y si logra indirectamente cierta credibilidad empírica.

Desde un punto de vista epistemológico, es relevante establecer si la formulación dialéctica satisface los motivos que llevaron a postularla. En este sentido, la apelación a procesos como la diferenciación e integración de los conceptos o la síntesis de las tensiones de las líneas cultural y natural ha mostrado ser adecuada para hacer inteligible a la novedad en el desarrollo psicológico. Finalmente, la caracterización de la dialéctica en Piaget y en Vigotsky como un enfoque metodológico común y con sus rasgos diferenciales en la explicación del desarrollo, tiene otro interés epistemológico: se ha dado un argumento sustentable a favor de la tesis que postula la compatibilidad entre los dos programas de investigación psicológica.

\section{Referencias}

Bidell, T. (1988). Vigotsky, Piaget and the Dialectic of Development. Human Development, 31, 329-348.

Boech, E. E. (1991). Symbolic act, theory and Cultural Psychology. Berlin, Germany: Sprenger-Verlag.

Bourdieu, P. (1988). Cosas dichas. Buenos Aires, Argentina: Gedisa.

Bourdieu, P., \& Waquant, L. (2005). El propósito de la Sociología Reflexiva. In P. Bourdieu \& L. Wacquant, Una invitación a la Sociología Reflexiva (pp. 105-205). Buenos Aires, Argentina: Siglo XXI.

Carey, S. (1999). Sources of conceptual change. In E. Scholnick \& K. Nelson (Eds.), Conceptual change. Piaget' legacy (pp. 293-326). London: Erlbaum.

Castorina, J. A., \& Baquero, R. (2005). Dialéctica y Psicología del Desarrollo. El pensamiento de Piaget y Vigotsky. Buenos Aires, Argentina: Amorrortu.

Deleuze, G. (1970). Diferencia y repetición. Buenos Aires, Argentina: Eudeba.

Elias, N. (1983). Compromiso y distanciamiento. Barcelona, España: Península.

García, R. (1970). Sobre la contradicción en la dialéctica de la naturaleza. In La explicación en las Ciencias. Barcelona, España: Martínez Roca.

García, R. (2002). El conocimiento en construcción. Barcelona, España: Gedisa.

Grüner, E. (2006). Lecturas culpables. Marx(ismos) y la praxis del conocimiento. In A. Borón, J. Amadeo, \& S. González (Eds.), La Teoría Marxista hoy. Problemas y perspectivas (pp. 105-147). Buenos Aires, Argentina: Clacso.

Inhelder, B., \& Piaget, J. (1979). Procedures et structures. Archives de Psychologie, 47, 165-176.

Lahire, B. (2006). El espíritu sociológico. Buenos Aires, Argentina: Manantial

Marx, C. (1971). El capital. México, DF: Fondo de Cultura Económica.

Overton, W. (1998). Developmental Psychology: Philosophy, concepts and methodology. In W. Damon (Series Ed.) \& R. Lerner (Vol. Ed.), Handbook of Child Psychology: Vol. 1. Theoretical models of human development $\left(5^{\text {th }}\right.$ ed., pp. $107-$ 187). New York: Wiley.

Piaget, J. (1969). El nacimiento de la inteligencia en el niño. Buenos Aires, Argentina: Aguilar.

Piaget, J. (1970). La psicología de la inteligencia. Buenos Aires, Argentina: Psique.

Piaget, J. (1978). Estudios sobre la contradicción. México, DF: Siglo XXI.

Piaget, J. (1981). Las formas elementales de la dialéctica. Barcelona, España: Gedisa.

Popper, K. (1967). El desarrollo del conocimiento científico. Buenos Aires, Argentina: Paidós.

Thelen, E., \& Smith, L. (1998). Dinamic Systems Theories. In W. Damon (Series Ed.) \& R. Lerner (Vol. Ed.), Handbook of Child Psychology: Vol. 1. Theoretical models of human development ( $5^{\text {th }}$ ed., pp. 563-633). New York: Wiley. 
Valsiner, J. (2000). Culture and human development. London: Sage.

Vigotsky, L. (1991a). El significado histórico de la crisis de la psicología: una investigación metodológica. In L. Vigotsky, Obras Escogidas: Vol. 1. Madrid, España: Visor. (Original work published 1927)

Vigotsky, L. (1991b). Sobre los sistemas psicológicos. In L. Vigotsky, Obras Escogidas: Vol. 1. Madrid, España: Visor. (Original work published 1930)

Vigotsky, L. (1993a). Obras Escogidas: Vol. 3. Historia del desarrollo de las funciones psíquicas superiores. Madrid, España: Visor. (Original work published 1931)

Vigotsky, L. (1993b). Pensamiento y lenguaje. In L. Vigotsky, Obras Escogidas: Vol. 2. Madrid, España: Visor. (Original work published 1934)

Vigotsky, L. (1994). Tkinking and concept formation in adolescente. In R. van der Veer \& J. Valsiner (Eds.), The Vygotsky reader. Oxford, UK: Blakwell. 